\title{
An Algorithm for Computing Continuous Chebyshev Approximations
}

\author{
By Zhongqi Jing and Adly T. Fam
}

\begin{abstract}
In this paper we introduce an algorithm for computing nonlinear continuous Chebyshev approximations. The algorithm is based on successive linearizations within adaptively adjusted neighborhoods. The convergence of the algorithm is proven under some general assumptions such that it is applicable for many Chebyshev approximation problems discussed in the literature. It, like the Remez exchange method, is purely continuous in the sense that it converges to a solution of a continuous Chebyshev approximation problem rather than one on a discretized set. Quadratic convergence is shown in so-called regular cases, including polynomial and nondegenerate rational approximations. We believe the algorithm is also computationally more efficient than some other algorithms. A few numerial examples are given to illustrate the basic features of the algorithm.
\end{abstract}

1. Introduction. In this paper we consider the following Chebyshev approximation problem. Find a parameter vector $A^{*}=\left(a_{1}^{*}, \ldots, a_{n}^{*}\right) \in \Omega$ to minimize $\max _{x \in \Delta} E(A, x)$, where $E(A, x)=|F(A, x)-f(x)|$ is the approximating error, $F(A, x)$ is an analytic function on $\Omega \times \Delta$ and $f(x)$ is piecewise analytic with, at most, a finite number of nonanalytic points. (We assume that if $x_{0}$ is a nonanalytic point, and $\left[x_{0}, x_{0}+\alpha\right) \subset \Delta$ for some $\alpha>0$, then a suitable redefinition of $f\left(x_{0}\right)$ (if necessary) would make $f$ representable by its Taylor series about $x_{0}$ in $\left[x_{0}, x_{0}+\tilde{\alpha}\right)$ for some $\tilde{\alpha}>0$, where the derivatives of $f$ at $x_{0}$ are replaced by derivatives from the right; we make the corresponding assumption if $\left(x_{0}-\alpha, x_{0}\right] \subset \Delta$ for some $\left.\alpha>0\right)$. $\Omega=\left\{Z=\left(z_{1}, \ldots, z_{n}\right)^{T} \mid L(Z) \leqslant 0\right\}$ is a bounded region in $n$-dimensional space, defined by a set of linear inequalities, $L(Z) \leqslant 0$. Because $\Omega$ can always be divided into a finite number of convex subregions, without loss of generality we will assume that $\Omega$ is convex. The set $\Delta$ is assumed to be the union of a finite number of closed intervals on the real axis. Thus, the problem involved is a continuous Chebyshev approximation problem.

The linear Chebyshev approximation can be found by the Remez exchange method [18], [4]. This method has been generalized to some other functions, including the important rational functions. However, difficulties due to the nonlinearity, the pole problem, the existence problem, and the degeneracy were encountered when the exchange method was applied to find the best approximation [18]. Several algorithms were suggested to partly overcome these difficulties; see, for example [18], [7]. It is expected that these difficulties appear in many more general nonlinear Chebyshev approximation problems. Watson described a method for

Received July 25, 1983, revised December 12, 1984, December 31, 1985, and May 6, 1986

1980 Mathematics Subject Classification. Primary 41A50, 65D15. 
calculating best nonlinear Chebyshev approximations [20]. However, it relied on the solution of a set of equations, which did not seem easy to solve except in a small neighborhood of the minimax point.

In this work we propose a different algorithm, which is designed to solve a wide class of continuous Chebyshev approximation problems, linear as well as nonlinear. For the approximating function $F(A, x)$ we only assume that it is analytic on $\Omega \times \Delta$ and the function to be approximated, $f(x)$, may be discontinuous as long as the local maxima of the approximating error function exist. The algorithm utilizes an iterative approach based on local linearizations. Like the multi-exchange method, at each iteration a discrete problem is solved on the set of points where the error function attains its local maxima. A technique of adaptively adjusting the linearization region is adopted to make the algorithm less sensitive to nonlinearity and degeneracy problems. A similar idea (but different scheme) was used by Madsen [13]. The general nonlinear Chebyshev approximation problem was treated by linearizing the approximating function in [5] and [9], but with somewhat different approaches.

The algorithm solves a bounded parameter approximation problem. Several authors have studied this problem, [17], [10] and [19]. To solve an unbounded parameter problem we can use a sufficiently large $\Omega$. Good choices of $\Omega$ and (if necessary) a suitable parameter mapping (i.e., introducing $A=g(W)$ and $F(A, x)$ $=F(g(W), x)=G(W, x)$ and applying the algorithm to the function $G$ on a set of parameters $W$ ) may exclude from $\Omega \times \Delta$ possible singular points of the approximating function $F(A, x)$. In many cases we simply choose $\Omega=\left\{Z=\left(z_{1}, \ldots, z_{n}\right)^{T}\right.$ : $\left.v_{i} \leqslant z_{i} \leqslant u_{i}, i=1, \ldots, n\right\}$, where the $v_{i}$ 's and $u_{i}$ 's are constants, if there is no specified $\Omega$.

The algorithm is proven to have sure convergence, and in so-called regular cases it converges quadratically. It is interesting to note that even though a descent method, in a neighborhood of $A^{*}$ it is equivalent to the multi-exchange method in linear regular cases, i.e., the two algorithms actually generate the same sequence that converges to $A^{*}$.

E. W. Cheney, G. D. Taylor, M. J. D. Powell, I. Barrodale and others studied computations of discrete Chebyshev approximations [1]-[4], [10]-[12], [17]. A continuous problem, then, can be solved by a discretization of $\Delta$. However, we sometimes encounter difficulties in choosing a suitable discretization point set in $\Delta$. In a nonlinear problem, the linear programming, or other methods involved in solving the discretized problem, are sensitive both in computational complexity and numerical behavior to the number of points in the set. In our proposed algorithm the point set is not fixed and usually contains only a small number of points. Working on this varying set, the algorithm converges directly to a stationary point, which is usually a local solution to a continuous Chebyshev approximation problem. The adaptive adjustment of linearization regions eliminates line searches in most of the iterations. All these things improve the efficiency of the algorithm.

We first describe the algorithm, with a brief discussion on its implementation, in Section 2. Then we prove the convergence theorem in Section 3. Some further discussions of its properties are given in Section 4. Several illustrative examples are presented in Section 5. 
2. The Algorithm. Before presenting the algorithm, we introduce some notation which is used throughout this paper. In the sequel we will assume that all local maxima of $E(A, x)$ with respect to $x$ for any fixed $A \in \Omega$ exist. By a local maximum $E\left(A, x^{*}\right)$ of $E(A, x)$ we mean that there exists an $\alpha>0$ such that $E(A, x) \leqslant E\left(A, x^{*}\right)$ for all $x \in\left[x^{*}-\alpha, x^{*}+\alpha\right] \cap \Delta$. The point $x^{*}$, then, is called a local maximum point. If the equality holds only for $x=x^{*}$, it is also called a strict one. A nonconstant analytic function can have only strict local maxima. $e(A)=$ $\max _{x \in \Delta} E(A, x)$ is called the maximum function of $E(A, x)$. The gradient vector with respect to $A$ and the inner product are respectively denoted by

$$
\nabla E(A, x)=\left(\frac{\partial E(A, x)}{\partial a_{1}}, \ldots, \frac{\partial E(A, x)}{\partial a_{n}}\right)^{T}
$$

and $(A, B)=\sum_{i=1}^{n} a_{i} b_{i}$. We use two norms, denoted by $\|A\|=\sqrt{(A, A)}$ and $\|A\|_{\infty}$ $=\max _{1 \leqslant i \leqslant n}\left|a_{i}\right|$. The notations of sets, $G(A, \delta)=\{Z \in \Omega \mid\|Z-A\| \leqslant \delta\}, G(x, \alpha)$ $=\{z \in \Delta|| z-x \mid \leqslant \alpha\}$ and $S(A, \delta)=\left\{Z \in \Omega \mid\|Z-A\|_{\infty} \leqslant \delta\right\}$ are also used frequently.

The objective of the algorithm is to find a sequence $\left\{A_{k}, k=0,1, \ldots\right\}$, starting with some initial approximation $A_{0}$ and a real positive number $d_{0}$, such that the sequence $\left\{e\left(A_{k}\right), k=0,1, \ldots\right\}$ either stops at or converges to a local minimum of $e(A)$. Suppose $A_{k}$ and $d_{k}$ have been found. Then $A_{k+1}$ and $d_{k+1}$ are generated by the following steps.

Step 1. Find all local maxima of $E(A, x), E\left(A_{k}, x_{i}\right), i=1,2, \ldots, N\left(A_{k}\right)$, with respect to $x$ for fixed $A=A_{k}$.

Step 2. Find $\overline{A_{k}}$ and $\bar{e}_{k}$ satisfying

$$
\begin{aligned}
\bar{e}_{k} & =\max _{1 \leqslant i \leqslant N\left(A_{k}\right)}\left[E\left(A_{k}, x_{i}\right)+\left(\nabla E\left(A_{k}, x_{i}\right), \overline{A_{k}}-A_{k}\right)\right] \\
& =\min _{A \in S\left(A_{k}, d_{k}\right)} \max _{1 \leqslant i \leqslant N\left(A_{k}\right)}\left[E\left(A_{k}, x_{i}\right)+\left(\nabla E\left(A_{k}, x_{i}\right), A-A_{k}\right)\right] .
\end{aligned}
$$

Step 3. If $\bar{e}_{k}=e\left(A_{k}\right), A_{k}$ is a stationary point of $e(A)$ and the algorithm is terminated. Otherwise, find the smallest nonnegative integer $L_{k}$ such that $A_{k+1}=$ $A_{k}+\left(\overline{A_{k}}-A_{k}\right) \gamma^{L_{k}}$ satisfies

$$
e\left(A_{k+1}\right) \leqslant e\left(A_{k}\right)-\beta_{1} \frac{\left\|A_{k+1}-A_{k}\right\|}{\left\|\overline{A_{k}}-A_{k}\right\|}\left(e\left(A_{k}\right)-\bar{e}_{k}\right),
$$

where $0<\gamma<1$ and $0<\beta_{1}<1$ are chosen constants.

Step 4. If

$$
\left|\bar{e}_{k}-e\left(\overline{A_{k}}\right)\right|>\beta_{2}\left(e\left(A_{k}\right)-\bar{e}_{k}\right) \text {, }
$$

set $d_{k+1}=\gamma_{1}\left\|\overline{A_{k}}-A_{k}\right\|_{\infty}$. Otherwise, set $d_{k+1}=\gamma_{2}\left\|\overline{A_{k}}-A_{k}\right\|_{\infty}$. Here $\beta_{2}>0, \gamma_{2}$ $>1,0<\gamma_{1}<1$ are chosen constants satisfying $\beta_{2} \geqslant \beta_{1}$ and $\beta_{2} \leqslant 1-\beta_{1}$.

Recalling the assumptions on $F(A, x)$ and $f(x)$ given earlier, we know that the first step defines a finite set of local maximum points, unless the error function $E\left(A_{k}, x\right)$ becomes a constant in some interval. We will assume that all $N\left(A_{k}\right)$, $k=0,1, \ldots$, are finite numbers and consequently Step 1 is well defined. Note that in normal approximation problems the above exceptional case is encountered very 
rarely. If at some $\tilde{A}, E(\tilde{A}, x)$ reduces to a constant, we can avoid this point either by removing it from $\Omega$ or by choosing an $A_{0}$ such that $e\left(A_{0}\right)<e(\tilde{A})$. Step 1 can be implemented by finding the zeros of $E_{x}^{\prime}\left(A_{k}, x\right)$ or by a one-dimensional search.

The second step is essentially a linear programming problem, since $S\left(A_{k}, d_{k}\right)$ is defined by linear inequalities. In the important special case where $\Omega=\{Z=$ $\left.\left(z_{1}, \ldots, z_{n}\right)^{T} \mid v_{i} \leqslant z_{i} \leqslant u_{i}\right\}$ is defined by a set of constants $u_{i}, v_{i}, i=1, \ldots, n$, $S\left(A_{k}, d_{k}\right)$ is simply $\left\{Z=\left(z_{1}, \ldots, z_{n}\right)^{T} \mid p_{i} \leqslant z_{i} \leqslant q_{i}\right\}$, where $p_{i}=\max \left(v_{i}, a_{k i}-d_{k}\right)$, $q_{i}=\min \left(u_{i}, a_{k i}+d_{k}\right)$, and $a_{k i}, i=1, \ldots, n$, are the components of $A_{k}$. By introducing a new set of variables $y_{i}=\left(\bar{a}_{k i}-p_{i}\right) /\left(q_{i}-p_{i}\right), i=1, \ldots, n$, and $y_{n+1}=f$ - $\bar{e}_{k}$, where $f=1.2 \max _{i} f_{i}, f_{i}=E\left(A_{k}, x_{i}\right)+\left(\nabla E\left(A_{k}, x_{i}\right), P-A_{k}\right), \quad P=$ $\left(p_{1}, \ldots, p_{n}\right)^{T}$, this step reduces to the following upper-bounded linear programming problem. Find $Y=\left(y_{1}, \ldots, y_{n+1}\right)^{T}$ to maximize $y_{n+1}$ subject to $Y \geqslant 0, y_{i} \leqslant 1$, $i=1, \ldots, n$, and $\sum_{j=1}^{n} C_{i j} y_{j}+y_{n+1} \leqslant g_{i}, i=1, \ldots, N\left(A_{k}\right)$, where

$$
C_{i j}=\partial E\left(A_{k}, x_{i}\right) / \partial a_{k j} \cdot\left(q_{j}-p_{j}\right)
$$

and $g_{i}=f-f_{i}$. With the initial basic feasible solution $Y=0$, this can be solved by the simplex method. An efficient modified simplex algorithm was developed in [8] to solve this upper-bounded problem.

The new point $A_{k+1}$ is found in Step 3. We will prove that there always exists an $L_{k}<\infty$ such that (2) holds, and (2) is sufficient to guarantee the convergence of the algorithm. In Step 4, $d_{k}$ which defines the size of the linearization region, is updated according to the linearization error.

The convergence of the algorithm is independent of the choice of the constants $\beta_{1}$, $\beta_{2}, \gamma, \gamma_{1}$ and $\gamma_{2}$ as long as they satisfy the conditions given in the algorithm. However, a judicious choice of them may improve the speed of convergence, especially when $A_{0}$ is far from $A^{*}$, and may reduce the sensitivity to numerical errors in the computation. Typical values we used in practice are $\beta_{1}=.01, \beta_{2}=.5$, $\gamma=.5, \gamma_{1}=.3$, and $\gamma_{2}=2$.

\section{The Convergence Theorem.}

Theorem 1. Let $A \in \Omega$ and $E\left(A, x_{a}\right)$ be a strict local maximum of $E(A, x)$. Then there exists an $\alpha>0$ such that the following is true: $E\left(A, x_{a}\right)=$ $\max _{x \in G\left(x_{a}, \alpha\right)} E(A, x)$, and for any given $\varepsilon>0$ there exists $a \theta>0$ such that for any $B, C=B+\Delta B \in G(A, \theta)$,

$$
\max _{x \in G\left(x_{a}, \alpha\right)} E(C, x) \leqslant \max _{x \in G\left(x_{a}, \alpha\right)} E(B, x)+\left(\nabla E\left(B, x_{b}\right), \Delta B\right)+\varepsilon\|\Delta B\|,
$$

where $x_{b} \in G\left(x_{a}, \alpha\right)$ is any local maximum point of $E(B, x)$.

Proof. We give only the proof for the case where $x_{a} \notin \partial \Delta$ and $f(x)$ is analytic in a neighborhood of $x_{a}$, since the proofs for other cases are similar. Let $m<\infty$ be the smallest positive integer such that $E_{x}^{(m)}\left(A, x_{a}\right) \neq 0$, where $E_{x}^{(m)}(\cdot, \cdot)$ denotes the $m$ th partial derivative with respect to $x$. $m$ must be even and $E_{x}^{(m)}\left(A, x_{a}\right)<0$. Thus, we are able to choose an $a>0$ and a $d>0$ such that $\left[x_{a}-a, x_{a}+a\right] \subset \Delta$ and $E(Z, x)$ is analytic on $S(A, d) \times G\left(x_{a}, a\right)$.

We first show that in a neighborhood of $\left(A, x_{a}\right)$, if $Z \rightarrow A$, then any local maximum point $x_{z}$ of $E(Z, x)$ approaches $x_{a}$. Let $Z \in S(A, d)$ and $x \in G\left(x_{a}, a\right)$. Then

$$
E(Z, x)=E(A, x)+(\nabla E(\hat{Z}, x), Z-A)
$$


where $\hat{Z} \in S(A, d)$ is some point dependent on $Z$ and

$$
E(A, x)=E\left(A, x_{a}\right)+\frac{\left(x-x_{a}\right)^{m}}{m !} E_{x}^{(m)}\left(A, x_{a}\right)+\frac{\left(x-x_{a}\right)^{m+1}}{(m+1) !} g(x),
$$

where $g(x)$ is some analytic function on $G\left(x_{a}, a\right)$. Further,

$$
\begin{aligned}
\frac{\partial E(Z, x)}{\partial x}= & \frac{\left(x-x_{a}\right)^{m-1}}{(m-1) !} E_{x}^{(m)}\left(A, x_{a}\right)+\frac{\left(x-x_{a}\right)^{m}}{m !} g(x) \\
& +\frac{\left(x-x_{a}\right)^{m+1}}{(m+1) !} g_{x}^{\prime}(x)+\left(\frac{\partial}{\partial x} \nabla E(\hat{Z}, x), Z-A\right) .
\end{aligned}
$$

Denote the upper bound of $\|\partial \nabla E(Z, x) / \partial x\|,(Z, x) \in S(A, d) \times G\left(x_{a}, a\right)$, by $M$ and choose $d \geqslant \delta>0, a \geqslant \alpha>0$ and $b>0$ such that $E\left(A, x_{a}\right)=$ $\max _{x \in G\left(x_{u}, \alpha\right)} E(A, x)$ and

(6) 1. $E\left(Z, x_{a} \pm \alpha\right)<\max _{x \in G\left(x_{a}, \alpha\right)} E(Z, x)$ for $Z \in G(A, \delta)$,

(7) 2. $E_{x}^{(m)}\left(A, x_{a}\right) \leqslant-b(m-1)$ !,

(8) 3. $\frac{\alpha}{m !}|g(x)| \leqslant \frac{b}{4}, \quad \frac{\alpha^{2}}{(m+1) !}\left|g_{x}^{\prime}(x)\right| \leqslant \frac{b}{4} \quad$ for $x \in G\left(x_{a}, \alpha\right)$.

Since $x_{z}$ is a local maximum point and (6) is assumed, $\partial E\left(Z, x_{z}\right) / \partial x=0$. Then, from (5), if $Z \in G(A, \delta), x_{z} \in G\left(x_{a}, \alpha\right)$,

$$
\begin{aligned}
\left(x_{z}\right. & \left.-x_{a}\right)^{m-1}\left[\frac{E_{x}^{(m)}\left(A, x_{a}\right)}{(m-1) !}+\frac{x_{z}-x_{a}}{m !} g\left(x_{z}\right)+\frac{\left(x_{z}-x_{a}\right)^{2}}{(m+1) !} g_{x}^{\prime}\left(x_{z}\right)\right] \\
& =-\left(\frac{\partial}{\partial x} \nabla E\left(\hat{Z}, x_{z}\right), Z-A\right) .
\end{aligned}
$$

In view of (7) and (8) we have

$$
\left|x_{z}-x_{a}\right|^{m-1} \leqslant \frac{2 M}{b}\|Z-A\| \text {. }
$$

We prove that with the above $\alpha$ the theorem holds. Suppose that $B, C \in G(A, \delta)$, $C=B+\Delta B$, and $x_{c}=x_{b}+\Delta x, x_{c} \in G\left(x_{a}, \alpha\right)$, is a local maximum point of $E(C, x)$. Then

$$
\begin{aligned}
\max _{x \in G\left(x_{a}, \alpha\right)} E(C, x) & =E\left(B+\Delta B, x_{c}\right) \\
& \leqslant \max _{x \in G\left(x_{a}, \alpha\right)} E(B, x)+\left(\nabla E\left(B, x_{c}\right), \Delta B\right)+o(\|\Delta B\|),
\end{aligned}
$$

where $\lim _{\|\Delta B\| \rightarrow 0} o(\|\Delta B\|) /\|\Delta B\|=0$. Consider the second term on the right-hand side,

$$
\begin{aligned}
\left(\nabla E\left(B, x_{c}\right), \Delta B\right) & =\left(\nabla E\left(B, x_{b}\right), \Delta B\right)+\Delta x\left(\frac{\partial}{\partial x} \nabla E\left(B, x_{b}+\eta \Delta x\right), \Delta B\right) \\
& \leqslant\left(\nabla E\left(B, x_{b}\right), \Delta B\right)+|\Delta x| \cdot M \cdot\|\Delta B\|,
\end{aligned}
$$

where $0 \leqslant \eta \leqslant 1$. Note that

$$
\begin{aligned}
|\Delta x| & =\left|\left(x_{c}-x_{a}\right)-\left(x_{b}-x_{a}\right)\right| \leqslant\left|x_{c}-x_{a}\right|+\left|x_{b}-x_{a}\right| \\
& \leqslant\left(\frac{2 M}{b}\right)^{1 /(m-1)}\left(\|B+\Delta B-A\|^{1 /(m-1)}+\|B-A\|^{1 /(m-1)}\right),
\end{aligned}
$$


where the last inequality holds because of (9). Thus, for any given $\varepsilon>0$, choose $0<\theta \leqslant \delta$ such that if $0<\beta \leqslant \theta$, then $o(\beta) / \beta \leqslant \varepsilon / 2$ and $(2 \theta M / b)^{1 /(m-1)} \cdot M \leqslant$ $\varepsilon / 4$. With this $\theta$, in view of (10), (11), and (12), (4) is true and the theorem is proven.

COROLlaRY 1. For sufficiently small $\Delta A$,

$$
\max _{x \in G\left(x_{a}, \alpha\right)} E(A+\Delta A, x)=E\left(A, x_{a}\right)+\left(\nabla E\left(A, x_{a}\right), \Delta A\right)+o(\|\Delta A\|) .
$$

Proof. This follows from (4) with $B=A, C=A+\Delta A$ and from the fact that

$$
\begin{aligned}
\max _{x \in G\left(x_{a}, \alpha\right)} E(A+\Delta A, x) & \geqslant E\left(A+\Delta A, x_{a}\right) \\
& =E\left(A, x_{a}\right)+\left(\nabla E\left(A, x_{a}\right), \Delta A\right)+o(\|\Delta A\|) .
\end{aligned}
$$

Recall that $\max _{x \in G\left(x_{a}, \alpha\right)} E(A, x)=E\left(A, x_{a}\right)$.

In the sequel we denote the set of all local maximum points of $E(A, x)$ by $X(A)$. We also need the notation

$$
X_{m}(A)=\{x \in X(A) \mid E(A, x)=e(A)\} .
$$

The maximum error $e(A)$ is continuous on $\Omega$, but not differentiable. To investigate its behavior in a neighborhood of $A$, we consider

$$
\lim _{Z \rightarrow A}(e(Z)-e(A)) /\|Z-A\|,
$$

where $Z \in \Omega$ approaches $A$ along a given straight line.

Definition 1. $e(A)$ is said to be directionally differentiable at $A$ if

$$
\lim _{\theta \rightarrow 0^{+}} \frac{e(A+\theta h)-e(A)}{\theta}
$$

exists for any $h \in H(A)$, where $H(A)=\{(Z-A) /\|Z-A\| \mid Z \in \Omega, Z \neq A\}$. Since $\Omega$ is a set defined by linear inequalities, there exists a $c(A)$ such that, for any $h \in H(A)$ and $0<\theta \leqslant c(A), A+\theta h \in \Omega$. The limit, denoted by $\partial e(A) / \partial h$, is called the derivative of $e(A)$ in direction $h$.

Lemma 1. $e(A)$ is continuous and directionally differentiable at any $A \in \Omega$. Moreover, for any $h \in H(A)$,

$$
\frac{\partial e(A)}{\partial h}=\max _{x \in X_{m}(A)}(\nabla E(A, x), h) .
$$

Proof. Let $x_{1}, \ldots, x_{r}$ be the elements of $X_{m}(A)$ and, for each $x_{i}$, let $\alpha_{i}$ be the number defined in Theorem 1 . Because of the continuity of $E(A, x)$, we are able to choose a $\delta>0, \delta \leqslant c(A)$, such that for any $Z \in G(A, \delta)$

$$
e(Z)=\max _{1 \leqslant i \leqslant r}\left[\max _{x \in G\left(x_{i}, \alpha_{i}\right)} E(Z, x)\right] \text {. }
$$

Then the continuity of $e(A)$ results from the fact that every term in the brackets is continuous at $Z=A$ according to Corollary 1 .

For $h \in H(A)$ and $\theta>0$ sufficiently small, in view of Corollary 1 ,

$$
\begin{aligned}
e(A+\theta h) & =\max _{1 \leqslant i \leqslant r}\left\{\max _{x \in G\left(x_{i}, \alpha_{i}\right)} E(A+\theta h, x)\right\} \\
& =\max _{1 \leqslant i \leqslant r}\left\{\max _{x \in G\left(x_{i}, \alpha_{i}\right)} E(A, x)+\left(\nabla E\left(A, x_{i}\right), \theta h\right)+o(\|\theta h\|)\right\} \\
& =e(A)+\max _{1 \leqslant i \leqslant r}\left[\theta\left(\nabla E\left(A, x_{i}\right), h\right)+o(\|\theta h\|)\right],
\end{aligned}
$$


where we used the fact that $\max _{x \in G\left(x_{i}, \alpha_{i}\right)} E(A, x)=e(A)$ for all $i$. Letting $\theta \rightarrow 0^{+}$, we obtain (14).

We define $A \in \Omega$ as a stationary point of $e(A)$ if $\min _{h \in H(A)} \partial e(A) / \partial h \geqslant 0$. It is clear that any local minimum point of $e(A)$ is a stationary point.

Denote, for $A \in \Omega$ and $d>0$,

$$
\begin{aligned}
\bar{e} & =\min _{Z \in S(A, d)} \max _{x \in X(A)}[E(A, x)+(\nabla E(A, x), Z-A)] \\
& =\max _{x \in X(A)}[E(A, x)+(\nabla E(A, x), \bar{A}-A)]
\end{aligned}
$$

and $D(A, d)=e(A)-\bar{e}$. Since $A \in S(A, d), D(A, d) \geqslant 0$.

Here $E(A, x)+(\nabla E(A, x), Z-A), Z \in S(A, d)$, is the linear approximation of the error function $E(A, x)$ in the neighborhood of $A, S(A, d)$, and (16) is the linearization of our minimax problem. Thus, in the neighborhood $S(A, d)$ the approximate solution by linearization is that at $\bar{A}$ the maximum of $E(A, x)$ with respect to $x$ obtains its minimum $\bar{e}$. For simplicity we do not explicitly indicate their dependence on $A$ and $d . \bar{e}$ and $\bar{A}$ can be viewed as the linearly approximate local minimax error and minimax point, respectively. $D(A, d)$, then, is the linear approximation of the local maximum descent of the maximum error $e(A)$.

LeMma 2. $A \in \Omega$ is a stationary point if and only if $D(A, d)=0$.

Proof. Assume $A$ is a stationary point of $e(A)$. If $\bar{A}=A$ then $D(A, d)=0$, so assume $\bar{A} \neq A$. Then $\max _{x \in X_{m}(A)}(\nabla E(A, x), \bar{h}) \geqslant 0$, where $\bar{h}=(\bar{A}-A) /\|\bar{A}-A\|$. Recalling that $e(A)=E(A, x)$ for $x \in X_{m}(A)$, and using the definition of (16), we obtain

$$
\begin{aligned}
\bar{e} & \geqslant \max _{x \in X_{m}(A)}[E(A, x)+(\nabla E(A, x), \bar{A}-A)] \\
& =e(A)+\|\bar{A}-A\| \max _{x \in X_{m}(A)}(\nabla E(A, x), \bar{h}) \geqslant e(A),
\end{aligned}
$$

i.e., $D(A, d) \leqslant 0$. Since $D(A, d) \geqslant 0$, we obtain $D(A, d)=0$.

Now suppose that $D(A, d)=0$. Assume, on the contrary, that $A$ is not a stationary point, i.e., there exists $h^{*} \in H(A)$ such that $\partial e(A) / \partial h^{*}=-\varepsilon$ for some $\varepsilon>0$. Let $\delta \leqslant \min (d, c(A))$ be such a positive number that for any $Z \in G(A, \delta)$

$$
\begin{aligned}
\max _{x \in X_{m}(A)}[E(A, x)+ & (\nabla E(A, x), Z-A)] \\
= & \max _{x \in X(A)}[E(A, x)+(\nabla E(A, x), Z-A)] .
\end{aligned}
$$

Then we have

$$
\begin{aligned}
\bar{e} & \leqslant \min _{Z \in G(A, \delta)} \max _{x \in X(A)}[E(A, x)+(\nabla E(A, x), Z-A)] \\
& =e(A)+\min _{Z \in G(A, \delta)} \max _{x \in X_{m}(A)}(\nabla E(A, x), Z-A) \\
& \leqslant e(A)+\delta \cdot \max _{x \in X_{m}(A)}\left(\nabla E(A, x), h^{*}\right)=e(A)-\delta \varepsilon,
\end{aligned}
$$

where we used the facts that $G(A, \delta) \subset S(A, d)$ and $A+\delta h^{*} \in G(A, \delta)$ and Eq. (14). This implies that $D(A, d) \geqslant \delta \varepsilon$, which contradicts our assumption and proves the lemma. 
THEOREM 2. The sequence $\left\{A_{k}\right\}$ generated by the algorithm either stops at a stationary point of $e(A)$ or must be an infinite sequence.

Proof. Recalling Step 3 in the algorithm, we must prove that, if $A_{k}$ is not a stationary point, then the algorithm does not stop and there always exists an $A_{k+1}$ satisfying (2). Since the first assertion follows directly from Lemma 2, we proceed to prove the second. Note that (2) can be rewritten as

$$
e\left(A_{k+1}\right) \leqslant e\left(A_{k}\right)-\beta_{1} \gamma^{L_{k}}\left(e\left(A_{k}\right)-\bar{e}_{k}\right) \text {. }
$$

To prove the existence of $A_{k+1}$, we show that, if $A \in \Omega$ is not a stationary point, then, for any $\beta_{1}>0, \beta_{1}<1$, and $d>0$, there exists an $a>0$ such that for any $\alpha>0, \alpha \leqslant a$,

$$
e(A)-e(A+\alpha(\bar{A}-A)) \geqslant \beta_{1} \alpha(e(A)-\bar{e})=\beta_{1} \alpha D(A, d),
$$

where $\bar{A}$ and $\bar{e}$, the linearly approximate local minimax point and minimax error, are defined in (16).

Since $A$ is not a stationary point, in view of Lemma $2, D(A, d)>0$. It follows from (16) and a deduction similar to (17), that $\max _{x \in X_{m}(A)}(\nabla E(A, x), \bar{A}-A) \leqslant$ $-D(A, d)$. Then, for sufficiently small $\alpha>0$, according to (15) and Corollary 1 ,

$$
\begin{aligned}
e(A+\alpha(\bar{A}-A)) & =\max _{1 \leqslant i \leqslant r} \max _{x \in G\left(x_{i}, \alpha_{i}\right)} E(A+\alpha(\bar{A}-A), x) \\
= & \max _{x \in X_{m}(A)}\left[E(A, x)+(\nabla E(A, x), \alpha(\bar{A}-A))+o_{x}(\alpha\|\bar{A}-A\|)\right] \\
= & e(A)+\max _{x \in X_{m}(A)}\left[(\nabla E(A, x), \alpha(\bar{A}-A))+o_{x}(\alpha\|\bar{A}-A\|)\right] \\
& \leqslant e(A)-\alpha D(A, d)+\max _{x \in X_{m}(A)} o_{x}(\alpha\|\bar{A}-A\|) .
\end{aligned}
$$

By choosing an $a>0$ such that for any $\alpha$ with $0<\alpha \leqslant a$

$$
\max _{x \in X_{m}(A)} o_{x}(\alpha\|\bar{A}-A\|) \leqslant\left(1-\beta_{1}\right) \alpha D(A, d),
$$

we obtain (19).

On the other hand, if $A_{k}$ is a stationary point, in view of Lemma 2, $D\left(A_{k}, d_{k}\right)=$ $e\left(A_{k}\right)-\bar{e}_{k}=0$ and the algorithm stops. The proof is complete.

Because the sequence $\left\{A_{k}\right\}$ lies in a compact set $\Omega$ and $\left\{e\left(A_{k}\right)\right\}$ is monotonically decreasing, $\left\{A_{k}\right\}$ must converge to a set of limit points. We assert that any of the limit points is a stationary point of $e(A)$. In order to present this result, we first show the following lemma.

Lemma 3. If $A^{*}$ is not a stationary point of $e(A)$, then there exist $\delta>0$ and $\varepsilon>0$ such that, for any $A \in G\left(A^{*}, \delta\right), D(A, d) \geqslant \varepsilon \min (d, \delta)$. If, moreover, $d \leqslant \delta$, then $\|\bar{A}-A\|_{\infty}=d$ and $|\bar{e}-e(\bar{A})| \leqslant \beta_{2} D(A, d)$, where $\beta_{2}$ is the constant defined in the algorithm.

Proof. Because $A^{*}$ is not a stationary point of $e(A)$, there exists an $h^{*} \in H\left(A^{*}\right)$ such that $\max _{x \in X_{m}\left(A^{*}\right)}\left(\nabla E\left(A^{*}, x\right), h^{*}\right)<0$. We first choose $\delta>0$ and $\varepsilon>0$ satisfying the following conditions.

(1) For any $A, Z \in S\left(A^{*}, 2 \delta\right), \max _{x \in X(A)}[E(A, x)+(\nabla E(A, x), Z-A)]$ is attained only at points in the set $R=G\left(x_{1}, \alpha_{1}\right) \cup G\left(x_{2}, \alpha_{2}\right) \cup \cdots \cup G\left(x_{r}, \alpha_{r}\right)$, where $x_{1}, \ldots, x_{r}$ are the elements of the set $X_{m}\left(A^{*}\right)$ and $\alpha_{i}, i=1, \ldots, r$, are 
respectively the numbers associated with $x_{i}, i=1, \ldots, r$, as defined in Theorem 1 . This is possible, since $\max _{x \in \Delta} E\left(A^{*}, x\right)$ is attained only at $x_{1}, \ldots, x_{r}$ (thus for $\left\|A-A^{*}\right\|$ sufficiently small, $\max _{x \in \Delta-R} E(A, x)<E\left(A, x_{i}\right)$ for $\left.i=1, \ldots, r\right)$ and, whenever $E(A, x) \neq 0, \quad \nabla E(A, x)=\operatorname{sgn}(F(A, x)-f(x)) \cdot \nabla F(A, x)$ (so $\left.\max _{x \in X(A)}|(\nabla E(A, x), Z-A)| \leqslant \max _{(B, x) \in \Omega \times \Delta}\|\nabla F(B, x)\| \cdot\|Z-A\|\right)$. Specifically, letting $Z=A$, we know that $\max _{x \in X(A)} E(A, x)$ is attained only at points in $R$ as well. This, then, implies that $\max _{x \in \Delta} E(A, x)=\max _{x \in X(A)} E(A, x)=$ $\max _{x \in R} E(A, x)$.

(2) $\left(\nabla E(A, x), h^{*}\right) \leqslant-\varepsilon$ for any $A \in G\left(A^{*}, \delta\right)$ and $x \in X(A) \cap R$. This is possible, since $E(A, x)$ is continuously differentiable and any $x \in X(A) \cap R$ approaches an element of $X_{m}\left(A^{*}\right)$ when $A \rightarrow A^{*}$. Refer to the proof of Theorem 1 .

(3) $A^{*}+2 g \in S\left(A^{*}, 2 \delta\right)$ for any $g$ satisfying $\|g\|_{\infty}=\delta$ and $g /\|g\| \in H\left(A^{*}\right)$. This is possible for sufficiently small $\delta$, because $\Omega$ is defined by a set of linear inequalities. It follows from this that, for any $A \in G\left(A^{*}, \delta\right), A+\delta h^{*} \in S(A, \delta)$.

(4) If $A \in G\left(A^{*}, \delta\right)$ and $\|\Delta A\|_{\infty} \leqslant \delta$, then for all $x_{i} \in X_{m}\left(A^{*}\right)$

$$
\max _{x \in G\left(x_{i}, \alpha_{i}\right)} E(A+\Delta A, x) \leqslant \max _{x \in G\left(x_{i}, \alpha_{i}\right)} E(A, x)+\left(\nabla E\left(A, x_{a i}\right), \Delta A\right)+\frac{\beta_{2} \varepsilon}{\sqrt{n}}\|\Delta A\|,
$$

where $x_{a i} \in G\left(x_{i}, \alpha_{i}\right) \cap X(A)$ and $E\left(A, x_{a i}\right)=\max _{x \in G\left(x_{i}, \alpha_{t}\right)} E(A, x)$. This is possible because of Theorem 1 .

(5) $E(A+\Delta A, x) \geqslant E(A, x)+(\nabla E(A, x), \Delta A)-\beta_{2} \varepsilon\|\Delta A\| / \sqrt{n}$, for any $x \in R$, $A \in G\left(A^{*}, \delta\right)$, and $\|\Delta A\|_{\infty} \leqslant \delta$. For sufficiently small $\delta$ this is possible, because $R$ and $G\left(A^{*}, \delta\right)$ are both compact sets and $E(A, x)$ is continuously differentiable.

Let us, then, prove that with the above $\delta$ and $\varepsilon$ the lemma is true. Consider any $A \in G\left(A^{*}, \delta\right)$. Notice that, in view of condition (3), $A+\min (\delta, d) \cdot h^{*} \in S(A, d)$, in view of condition (1), $\max _{x \in X(A)}$ can be replaced by $\max _{x \in X(A) \cap R}$ in the next equation and, consequently, condition (2) can be adopted. Thus

$$
\begin{aligned}
\bar{e} & =\min _{Z \in S(A, d)} \max _{x \in X(A)}[E(A, x)+(\nabla E(A, x), Z-A)] \\
& \leqslant \max _{x \in X(A)}\left[E(A, x)+\min (\delta, d) \cdot\left(\nabla E(A, x), h^{*}\right)\right] \leqslant e(A)-\varepsilon \cdot \min (\delta, d) .
\end{aligned}
$$

The first assertion is proven. To show the second assertion let us assume, on the contrary, that $\|\bar{A}-A\|_{\infty}<d$. Then, in view of condition (3), there exists $\eta>0$ such that $\bar{A}+\eta h^{*} \in S(A, d)$. By the definition of $\bar{A}$,

$$
\begin{aligned}
\max _{x \in X(A)}[E( & A, x)+(\nabla E(A, x), \bar{A}-A)] \\
& \leqslant \max _{x \in X(A)}\left[E(A, x)+\left(\nabla E(A, x), \bar{A}+\eta h^{*}-A\right)\right] \\
& =E(A, \tilde{x})+(\nabla E(A, \tilde{x}), \bar{A}-A)+\left(\nabla E(A, \tilde{x}), \eta h^{*}\right),
\end{aligned}
$$

where, since $A, \bar{A}+\eta h^{*} \in S\left(A^{*}, 2 \delta\right)$, we have $\tilde{x} \in X(A) \cap R$. Because the sum of the first two terms on the right-hand side is less than or equal to the expression on the left-hand side, $\left(\nabla E(A, \tilde{x}), \eta h^{*}\right) \geqslant 0$. This, however, contradicts our condition (2). Thus $\|\bar{A}-A\|_{\infty} \geqslant d$. Because of the obvious observation that $\|\bar{A}-A\|_{\infty} \leqslant d$, we know that $\|\bar{A}-A\|_{\infty}=d$. 
Let $\Delta A=\bar{A}-A$. Because of the conditions (1) and (4) and the fact that $\bar{A} \in S\left(A^{*}, 2 \delta\right)$

$$
\begin{aligned}
e(\bar{A}) & =\max _{x \in R} E(A+\Delta A, x) \\
& \leqslant \max _{1 \leqslant i \leqslant r}\left[\max _{x \in G\left(x_{i}, \alpha_{i}\right)} E(A, x)+\left(\nabla E\left(A, x_{a i}\right), \Delta A\right)+\frac{\beta_{2} \varepsilon}{\sqrt{n}}\|\Delta A\|\right] \\
& =\max _{1 \leqslant i \leqslant r}\left[E\left(A, x_{a i}\right)+\left(\nabla E\left(A, x_{a i}\right), \Delta A\right)\right]+\frac{\beta_{2} \varepsilon}{\sqrt{n}}\|\Delta A\| \leqslant \bar{e}+\frac{\beta_{2} \varepsilon}{\sqrt{n}}\|\Delta A\|,
\end{aligned}
$$

where the last step results from (16) and the fact that $\left\{x_{a i}, i=1, \ldots, r\right\} \subset X(A)$. In view of

$$
D(A, d) \geqslant \varepsilon d=\varepsilon\|\Delta A\|_{\infty} \geqslant \varepsilon \frac{\|\Delta A\|}{\sqrt{n}},
$$

we have

$$
e(\bar{A})-\bar{e} \leqslant \beta_{2} D(A, d) .
$$

On the other hand, according to the conditions (1) and (5),

$$
\begin{aligned}
e(\bar{A}) & =\max _{x \in R} E(A+\Delta A, x) \\
& \geqslant \max _{x \in R}[E(A, x)+(\nabla E(A, x), \Delta A)]-\frac{\beta_{2} \varepsilon}{\sqrt{n}}\|\Delta A\| \\
& \geqslant \bar{e}-\frac{\beta_{2} \varepsilon}{\sqrt{n}}\|\Delta A\| \geqslant \bar{e}-\beta_{2} D(A, d),
\end{aligned}
$$

where we used (16). The last step follows from (21). The last assertion of the lemma, then, is the combination of (22) and (23).

THEOREM 3. Any limit point of the sequence $\left\{A_{i}, i=0,1, \ldots\right\}$, generated by the algorithm, is a stationary point of $e(A)$.

Proof. We consider two separate cases.

Case 1. $\left\{A_{i}\right\}$ converges to a single limit point $A^{*}$. We must show that $A^{*}$ is a stationary point. Assume, on the contrary, that $A^{*}$ is not. Let $\delta$ and $\varepsilon$ be the numbers defined in Lemma 3 at $A^{*}$. In view of the continuity of $e(A)$, there exists a $K>0$ such that, for any $i \geqslant K, A_{i} \in G\left(A^{*}, \delta / 2 \gamma_{2}\right)$ and $\left|e\left(A_{i}\right)-e\left(A^{*}\right)\right| \leqslant \frac{1}{2} \beta_{1} \delta \varepsilon$, where $\gamma_{2}$ and $\beta_{1}$ are the constants in the algorithm.

We assert that there exists a $k \geqslant K$ such that $\delta / \gamma_{2}<d_{k} \leqslant \delta$.

If $d_{K} \leqslant \delta / \gamma_{2}$, according to Lemma 3 and Step 4 of the algorithm, $d_{K+1}=\gamma_{2} d_{K}$. If, again, $d_{K+1} \leqslant \delta / \gamma_{2}$, then $d_{K+2}=\gamma_{2} d_{K+1}$. Thus, since $\gamma_{2}>1$, we have $d_{k}>\delta / \gamma_{2}$ for some $k \geqslant K$.

Suppose that $d_{K}>\delta$. Lemma 3 , then, indicates $D\left(A_{K}, d_{K}\right) \geqslant \varepsilon \delta$. We note that $A_{K+1} \neq \bar{A}_{K}$, i.e., $L_{K} \neq 0$, where $L_{K}$ is the integer found in Step 3 of the algorithm. This is because if, otherwise, $A_{K+1}=\bar{A}_{K}$, then in view of the algorithm,

$$
e\left(A_{K}\right)-e\left(A_{K+1}\right) \geqslant \beta_{1}\left(e\left(A_{K}\right)-\bar{e}_{K}\right) \geqslant \beta_{1} \varepsilon \delta,
$$


which contradicts our assumption that $\left|e\left(A_{i}\right)-e\left(A^{*}\right)\right| \leqslant \frac{1}{2} \beta_{1} \delta \varepsilon$, for all $i \geqslant K$. Thus $e\left(A_{K}\right)-e\left(\bar{A}_{K}\right)<\beta_{1} D\left(A_{K}, d_{K}\right)$. Recalling that $1-\beta_{1} \geqslant \beta_{2}$, we have

$$
\begin{aligned}
e\left(\bar{A}_{K}\right)-\bar{e}_{K} & >e\left(A_{K}\right)-\bar{e}_{K}-\beta_{1} D\left(A_{K}, d_{K}\right) \\
& =\left(1-\beta_{1}\right) D\left(A_{K}, d_{K}\right) \geqslant \beta_{2} D\left(A_{K}, d_{K}\right) .
\end{aligned}
$$

Since the right-hand side is nonnegative, we further obtain $\left|e\left(\bar{A}_{K}\right)-\bar{e}_{K}\right|>$ $\beta_{2} D\left(A_{K}, d_{K}\right)$. Then, in terms of the algorithm, $d_{K+1}=\gamma_{1}\left\|\bar{A}_{K}-A_{K}\right\|_{\infty} \leqslant \gamma_{1} d_{K}$. If, again, $d_{K+1}>\delta$, we have $d_{K+2} \leqslant \gamma_{1} d_{K+1}$. By repeating the same argument, in view of $\gamma_{1}<1$, we know that there exists $\tilde{k} \geqslant K$ such that $d_{\tilde{k}}<\delta$. Then, applying, if necessary, our previous discussion for the case where $d_{K} \leqslant \delta / \gamma_{2}$, we show the existence of $k$.

Now we prove that, if $d_{k} \leqslant \delta$, then $A_{k+1}=\overline{A_{k}}$. It follows from Lemma 3 that $\bar{e}_{k}-e\left(\bar{A}_{k}\right) \geqslant-\beta_{2}\left(e\left(A_{k}\right)-\bar{e}_{k}\right)$, i.e.,

$$
e\left(\overline{A_{k}}\right) \leqslant e\left(A_{k}\right)-\left(1-\beta_{2}\right)\left(e\left(A_{k}\right)-\bar{e}_{k}\right) \leqslant e\left(A_{k}\right)-\beta_{1}\left(e\left(A_{k}\right)-\bar{e}_{k}\right),
$$

where we used the fact that $D\left(A_{k}, d_{k}\right)=e\left(A_{k}\right)-\bar{e}_{k} \geqslant 0$. Then, in terms of Step 3 of the algorithm, $A_{k+1}=\overline{A_{k}}$.

Finally, according to Lemma 3 , we have $\left\|A_{k+1}-A_{k}\right\| \geqslant\left\|A_{k+1}-A_{k}\right\|_{\infty}=$ $\left\|\overline{A_{k}}-A_{k}\right\|_{\infty}=d_{k}>\delta / \gamma_{2}$. However, this is impossible because both $A_{k+1}$ and $A_{k}$ are in $G\left(A^{*}, \delta / 2 \gamma_{2}\right)$. Hence $A^{*}$ must be a stationary point of $e(A)$.

Case 2. There is more than one limit point of $\left\{A_{i}\right\}$. Let $A^{*}$ be any of these limit points. To prove that $A^{*}$ is a stationary point, we again assume, on the contrary, that $A^{*}$ is not a stationary point and $\delta$ and $\varepsilon$ are the numbers defined in Lemma 3 for $A^{*}$. We first show that, if $A_{i} \in G\left(A^{*}, \delta\right)$, then

$$
e\left(A_{i}\right)-e\left(A_{i+1}\right) \geqslant \beta_{1} \varepsilon \cdot \min \left(\frac{1}{\sqrt{n}}, \frac{\delta}{S}\right) \cdot\left\|\Delta A_{i}\right\|,
$$

where $\Delta A_{i}=A_{i+1}-A_{i}$ and $S=\max _{Z_{1}, Z_{2} \in \Omega}\left\|Z_{1}-Z_{2}\right\|$. Because $\Omega$ is a bounded region, $S<\infty$.

If $d_{i} \leqslant \delta$, then $A_{i+1}=\bar{A}_{i}$, a fact shown in the first part of the proof, and, consequently,

$$
\begin{aligned}
e\left(A_{i}\right)-e\left(A_{i+1}\right) & =e\left(A_{i}\right)-e\left(\overline{A_{i}}\right) \geqslant \beta_{1}\left(e\left(A_{i}\right)-\bar{e}_{i}\right) \\
& =\beta_{1} D\left(A_{i}, d_{i}\right) \geqslant \beta_{1} \varepsilon d_{i} \geqslant \beta_{1} \varepsilon\left\|\Delta A_{i}\right\| / \sqrt{n},
\end{aligned}
$$

where (24) and Lemma 3 were used.

If $d_{i}>\delta$, in view of the third step of the algorithm,

$$
e\left(A_{i}\right)-e\left(A_{i+1}\right) \geqslant \beta_{1} \frac{\left\|A_{i+1}-A_{i}\right\|}{\left\|\overline{A_{i}}-A_{i}\right\|} D\left(A_{i}, d_{i}\right) \geqslant \beta_{1} \frac{\left\|\Delta A_{i}\right\|}{S} \cdot \varepsilon \delta .
$$

Hence, (25) is always true.

Let $r=\left\|A^{*}-\tilde{A}\right\|$, where $\tilde{A} \neq A^{*}$ is any of the other limit points, $\eta=\min (\delta, r / 2)$ and $\theta \leqslant \eta / 2$ be a positive number satisfying that for any $A \in G\left(A^{*}, \theta\right)$

$$
\left|e(A)-e\left(A^{*}\right)\right|<\frac{1}{4} \varepsilon \beta_{1} \eta \cdot \min \left(\frac{1}{\sqrt{n}}, \frac{\delta}{S}\right) .
$$


Consider $i<j<k$ such that $A_{i}, A_{k} \in G\left(A^{*}, \theta\right), A_{i}, A_{i+1}, \ldots, A_{j-1} \in G\left(A^{*}, \eta\right)$ and $A_{j} \notin G\left(A^{*}, \eta\right)$. Since $A^{*}$ and $\tilde{A}$ are both limit points, such $i, j$, and $k$ exist. Recalling that $\left\{e\left(A_{i}\right)\right\}$ is a monotonically decreasing sequence, we have $e\left(A_{i}\right)>$ $e\left(A_{j}\right)>e\left(A_{k}\right)$. This and (26) mean that

$$
e\left(A_{i}\right)-e\left(A_{j}\right)<e\left(A_{i}\right)-e\left(A_{k}\right)<\frac{1}{2} \varepsilon \beta_{1} \eta \cdot \min \left(\frac{1}{\sqrt{n}}, \frac{\delta}{S}\right) .
$$

On the other hand, in view of (25),

$$
\begin{aligned}
e\left(A_{i}\right)-e\left(A_{j}\right) & =\sum_{m=i}^{j-1}\left(e\left(A_{m}\right)-e\left(A_{m+1}\right)\right) \\
& \geqslant \beta_{1} \varepsilon \cdot \min \left(\frac{1}{\sqrt{n}}, \frac{\delta}{S}\right) \sum_{m=i}^{j-1}\left\|\Delta A_{m}\right\| \geqslant \frac{1}{2} \eta \beta_{1} \varepsilon \cdot \min \left(\frac{1}{\sqrt{n}}, \frac{\delta}{S}\right),
\end{aligned}
$$

where we used that $\sum_{m=i}^{j-1}\left\|\Delta A_{m}\right\| \geqslant\left\|A_{j}-A_{i}\right\| \geqslant \eta / 2$. Being absurd, this completes the proof.

4. Discussion. The convergence rate depends on local properties in a neighborhood of a stationary point. In this section we define a special case, called the regular case, and show that in the regular case the algorithm converges quadratically. We then show that the algorithm reduces to the multi-exchange method in a regular linear approximation problem satisfying the Haar condition.

Definition 2. Let $A^{*} \in \Omega$ be a minimax point of $E(A, x)$, i.e., $e(A)$ attains its minimum at $A^{*}$. $A^{*}$ is called a regular minimax point if the following hold:

(a) There are exactly $n+1$ elements in the set $X_{m}\left(A^{*}\right)$ and, moreover, for each $x_{i} \in X_{m}\left(A^{*}\right)$ either $f(x)$ is analytic in $\left(x_{i}-\alpha, x_{i}+\alpha\right)$ for some $\alpha>0$ and $E_{x}^{\prime \prime}\left(A^{*}, x_{i}\right) \neq 0$, or there exists $\alpha>0$ such that for any $A \in \Omega$ with sufficiently small $\left\|A-A^{*}\right\|, x_{i}$ is the only local maximum point of $E(A, x)$ in $G\left(x_{i}, \alpha\right)$.

(b) The minimum directional derivative of the maximum error function $e(A)$ is positive at $A^{*}$, i.e.,

$$
\min _{g \in H\left(A^{*}\right)} \frac{\partial e\left(A^{*}\right)}{\partial g}>0
$$

It follows from the above definition that for $\tilde{\delta}>0$ and $\tilde{\alpha}>0$ sufficiently small, and for any $x_{i} \in X_{m}\left(A^{*}\right)$, if $A \in G\left(A^{*}, \tilde{\delta}\right)$ then there is exactly one local maximum point $x_{i}(A)$ of $E(A, x)$ in $G\left(x_{i}, \tilde{\alpha}\right)$. To see this, note that if $\tilde{\delta}>0$ and $\tilde{\alpha}>0$ are sufficiently small and $E_{x}^{\prime \prime}\left(A^{*}, x\right) \neq 0$, then we have $E_{x}^{\prime \prime}(A, x) \neq 0$ for all $(A, x) \in$ $G\left(A^{*}, \tilde{\delta}\right) \times G\left(x_{i}, \tilde{\alpha}\right)$ (so $E_{x}^{\prime}(A, x)$ has only one zero in $G\left(x_{i}, \tilde{\alpha}\right)$ ). Now for $A \in$ $G\left(A^{*}, \tilde{\delta}\right)$, we can define functions

$$
f_{i}(A)=E\left(A, x_{i}(A)\right), \quad i=1, \ldots, n+1 .
$$

For some $\delta$ with $0<\delta \leqslant \tilde{\delta}$ we have for every $A \in G\left(A^{*}, \delta\right)$ that $e(A)=\max _{i} f_{i}(A)$, $f_{i}$ is analytic at $A$ for all $i$, and for all $i$

$$
\nabla f_{i}(A)=\nabla E\left(A, x_{i}(A)\right)
$$

where on the right-hand side the partial derivatives are taken with $x_{i}(A)$ fixed. The first assertion in the last sentence follows from arguments similar to those of condition (1) in Lemma 3. The analyticity of $f_{i}$ at $A$ for $\left\|A-A^{*}\right\|$ sufficiently small 
holds because either the Implicit Function Theorem (applied to the equation $\left.E_{x}^{\prime}(A, x)=0\right)$ implies $\nabla x_{i}(A)=-\nabla E_{x}^{\prime}\left(A, x_{i}(A)\right) / E_{x}^{\prime \prime}\left(A, x_{i}(A)\right)$, which implies the analyticity of $x_{i}(A)$ (and thus of $f_{i}(A)=E\left(A, x_{i}(A)\right)$ ), or $x_{i}(A)$ is fixed at $x_{i}$, and $f_{i}(A)=E\left(A, x_{i}\right)$ is analytic. Finally, (29) follows either from the chain rule, or from the fact that $x_{i}(A)$ is fixed at $x_{i}$.

The following properties of a regular minimax point will be used later.

LEMma 4. Let $A^{*}$ be the above regular minimax point. Then there exists an $\varepsilon$-neighborhood $G\left(A^{*}, \varepsilon\right), 0<\varepsilon \leqslant \delta$, such that for any $A \in G\left(A^{*}, \varepsilon\right)$

$$
\text { (1) } C_{M}\left\|A-A^{*}\right\| \geqslant\left|e(A)-e\left(A^{*}\right)\right| \geqslant C \cdot\left\|A-A^{*}\right\| \text {, }
$$

where $C_{M}>0$ and $C>0$ are some constants;

(2) if $A^{*}$ is not on the boundary of $\Omega$, the matrix

$$
J(A)=\left[\begin{array}{cccc}
\frac{\partial f_{1}(A)}{\partial a_{1}} & \cdots & \frac{\partial f_{1}(A)}{\partial a_{n}} & -1 \\
\frac{\partial f_{n+1}(A)}{\partial a_{1}} & \cdots & \frac{\partial f_{n+1}(A)}{\partial a_{n}} & -1
\end{array}\right]
$$

is of full rank;

(3) for sufficiently large $d$, the solution $\bar{A}$ in the linear programming problem (16) is determined by the intersection of the $n+1$ tangent hyperplanes of $f_{i}(A), i=1, \ldots, n$ +1 , at $A$, i.e, if either $A^{\prime} \in S(A, d)$ (where $A^{\prime}$ denotes the intersection point) or $\|\bar{A}-A\|_{\infty}<d$, then $\bar{A}=A^{\prime}$

(4) if $A, A^{\prime} \in G\left(A^{*}, \varepsilon\right)$, then

$$
\left|e\left(A^{\prime}\right)-e\right| \leqslant \frac{C}{3\left(1+1 / \beta_{2}\right)}\left\|A^{\prime}-A\right\|,
$$

where $A^{\prime}$ is defined in (3), $e=f_{i}(A)+\left(\nabla f_{i}(A), A^{\prime}-A\right)$ for any $1 \leqslant i \leqslant n+1$ and $\beta_{2}$ is the constant used in the algorithm. Specifically, if $\bar{A}=A^{\prime}$, then $\bar{e}=e$.

Proof. (1) As the first relation in (30) follows from (15) and Corollary 1, we proceed to show the second. In view of (27), let $\min _{h \in H\left(A^{*}\right)} \partial e\left(A^{*}\right) / \partial h \geqslant C_{1}$, where $C_{1}>0$ is some constant. Recalling Lemma 1 and Eq. (29), we have $\partial e\left(A^{*}\right) / \partial g=$ $\left(\nabla f_{i}\left(A^{*}\right), g\right) \geqslant C_{1}$ for any $g \in H\left(A^{*}\right)$, where $i$ is a number such that

$$
\left(\nabla E\left(A^{*}, x_{i}\right), g\right)=\max _{1 \leqslant j \leqslant n+1}\left(\nabla E\left(A^{*}, x_{j}\right), g\right) \text {. }
$$

Then, if $A=A^{*}+\operatorname{tg} \in G\left(A^{*}, \delta\right), t>0$,

$$
\begin{aligned}
e(A)-e\left(A^{*}\right) & \geqslant f_{i}(A)-e\left(A^{*}\right)=\left(\nabla f_{i}\left(A^{*}\right), A-A^{*}\right)+o\left(\left\|A-A^{*}\right\|\right) \\
& \geqslant C_{1}\left\|A-A^{*}\right\|+o\left(\left\|A-A^{*}\right\|\right) .
\end{aligned}
$$

Choose $C=C_{1} / 2$. Then for each $g \in H\left(A^{*}\right)$ there exists a maximal $r(g)>0$, $r(g) \leqslant \delta$, such that if $A$ satisfies $\left(A-A^{*}\right) /\left\|A-A^{*}\right\|=g$ and $\left\|A-A^{*}\right\| \leqslant r(g)$, then (30) is true. Denote $\varepsilon_{1}=\min \left(\bar{\varepsilon}_{1}, \inf _{g \in H\left(A^{*}\right)} r(g)\right)$, where $\bar{\varepsilon}_{1}>0$ is sufficiently small to guarantee the first relation in (30). Then (30) holds for any $A \in G\left(A^{*}, \varepsilon_{1}\right)$.

(2) Assume rank $J\left(A^{*}\right)<n+1$. Then there exists a vector $B=\left(b_{1}, \ldots, b_{n+1}\right)^{T} \neq$ 0 such that $b_{n+1} \leqslant 0, \sum_{i=1}^{n} b_{i}^{2}=1$ and $J\left(A^{*}\right) \cdot B=0$, i..e, $\left(\nabla E\left(A^{*}, x_{i}\right), B_{n}\right)=b_{n+1}$ for $i=1, \ldots, n+1$, where $B_{n}=\left(b_{1}, \ldots, b_{n}\right)^{T}$. This means that $\partial e\left(A^{*}\right) / \partial B_{n}=b_{n+1}$ $\leqslant 0$. Since $A^{*}$ is not on the boundary of $\Omega, B_{n} \in H\left(A^{*}\right)$. This, however, contradicts 
(27) and proves that $\operatorname{rank} J\left(A^{*}\right)=n+1$. Because $f_{i}(A), i=1, \ldots, n+1$, are continuously differentiable for $A \in G\left(A^{*}, \delta\right)$, there exists an $\varepsilon_{2}>0, \varepsilon_{2} \leqslant \delta$, such that rank $J(A)=n+1$ for all $A \in G\left(A^{*}, \varepsilon_{2}\right)$.

(3) The fact that $\operatorname{rank} J(A)=n+1, A \in G\left(A^{*}, \varepsilon_{2}\right)$, indicates that the $n+1$ tangent hyperplanes of $f_{i}(A), i=1, \ldots, n+1$, at $A$ intersect at a unique point $A^{\prime}$. We show that if $d$ is large enough such that $A^{\prime} \in S(A, d)$ and $A$ is close to $A^{*}$, then $\bar{A}=A^{\prime}$. We first claim that for any $d>0$, if $\left\|A-A^{*}\right\|$ is sufficiently small, then

$$
\bar{e}=\min _{Z \in S(A, d)} \max _{1 \leqslant i \leqslant n+1}\left[E\left(A, x_{i}(A)\right)+\left(\nabla E\left(A, x_{i}(A)\right), Z-A\right)\right],
$$

i.e., the maximum in (16) occurs at some $x_{i}(A)$. To see this, suppose $A_{j} \rightarrow A^{*}$, and let $Z_{j} \in S\left(A_{j}, d\right)$ satisfy

$$
\begin{gathered}
\min _{Z \in S\left(A_{j}, d\right)} \max _{x \in X\left(A_{j}\right)}\left[E\left(A_{j}, x\right)+\left(\nabla E\left(A_{j}, x\right), Z-A_{j}\right)\right] \\
=\max _{x \in X\left(A_{j}\right)}\left[E\left(A_{j}, x\right)+\left(\nabla E\left(A_{j}, x\right), Z_{j}-A_{j}\right)\right] .
\end{gathered}
$$

We assert that $Z_{j} \rightarrow A^{*}$, for if not, suppose (going to subsequences if necessary) $Z_{j} \rightarrow Z^{*} \in S\left(A^{*}, d\right)$; then by Lemma 1 and Definition 2 we have for some $i$ that $E\left(A^{*}, x_{i}\right)+\left(\nabla E\left(A^{*}, x_{i}\right), Z^{*}-A^{*}\right)>e\left(A^{*}\right)$, so for $j$ sufficiently large

$$
E\left(A_{j}, x_{i}\left(A_{j}\right)\right)+\left(\nabla E\left(A_{j}, x_{i}\left(A_{j}\right)\right), Z_{j}-A_{j}\right)>e\left(A_{j}\right),
$$

violating the choice of $Z_{j}$. The claim now follows from part (1) of Lemma 3 and the paragraph following Definition 2. Thus for some $\bar{\varepsilon}_{2}$ with $0<\bar{\varepsilon}_{2} \leqslant \varepsilon_{2}$, if $\left\|A-A^{*}\right\|$ $\leqslant \bar{\varepsilon}_{2}$ we have that

$$
\begin{aligned}
\bar{e} & =\min _{Z \in S(A, d)} \max _{1 \leqslant i \leqslant n+1}\left[f_{i}(A)+\left(\nabla f_{i}(A), Z-A\right)\right] \\
& =\max _{1 \leqslant i \leqslant n+1}\left[f_{i}(A)+\left(\nabla f_{i}(A), \bar{A}-A\right)\right] .
\end{aligned}
$$

The tangent hyperplane of $f_{i}(A)$ at $A$ is written as $y_{i}=f_{i}(A)+\left(\nabla f_{i}(A), Z-A\right)=$ $e+\left(\nabla f_{i}(A), Z-A^{\prime}\right), Z \in R^{n}$, where $e=f_{i}(A)+\left(\nabla f_{i}(A), A^{\prime}-A\right)$ for all $i$.

Because of (27), Lemma 1 and (29), for any $g \in H\left(A^{*}\right)$,

$$
\max _{1 \leqslant i \leqslant n+1}\left(\nabla E\left(A^{*}, x_{i}\right), g\right)=\max _{1 \leqslant i \leqslant n+1}\left(\nabla f_{i}\left(A^{*}\right), g\right)>0 .
$$

Since $f_{i}(A), i=1, \ldots, n+1$, are continuously differentiable on $G\left(A^{*}, \delta\right)$, there exists an $\varepsilon_{3}>0, \varepsilon_{3} \leqslant \bar{\varepsilon}_{2}$, such that, for all $A \in G\left(A^{*}, \varepsilon_{3}\right)$,

$$
\max _{1 \leqslant i \leqslant n+1}\left(\nabla f_{i}(A), g\right)>0 \text { for all } g \in H\left(A^{*}\right) \text {. }
$$

Thus, for any $A \in G\left(A^{*}, \varepsilon_{3}\right), \min _{Z \in S(A, d)} \max _{1 \leqslant i \leqslant n+1}\left[e+\left(\nabla f_{i}(A), Z-A^{\prime}\right)\right]$ is attained at $Z=A^{\prime}$, i.e., $\bar{A}=A^{\prime}$, if $A^{\prime} \in S(A, d)$. Further, if $A^{\prime} \notin S(A, d)$ then $\|\bar{A}-A\|_{\infty}=d$, so if $\|\bar{A}-A\|_{\infty}<d$ then $A^{\prime} \in S(A, d)$ and so $\bar{A}=A^{\prime}$.

(4) We can assume $\varepsilon_{3}$ is so small that $A^{\prime} \in G\left(A^{*}, \varepsilon_{3}\right)$ implies $e\left(A^{\prime}\right)=f_{i}\left(A^{\prime}\right)$ for some $i$. Then $e=f_{i}(A)+\left(\nabla f_{i}(A), A^{\prime}-A\right)$, because $A^{\prime}$ is the intersection of $n+1$ tangent hyperplanes. Let $\varepsilon_{4}>0$ be a number such that for any $A, Z \in G\left(A^{*}, \varepsilon_{4}\right)$ and $1 \leqslant i \leqslant n+1$,

$$
\left|f_{i}(Z)-\left(f_{i}(A)+\left(\nabla f_{i}(A), Z-A\right)\right)\right| \leqslant \frac{C}{3\left(1+1 / \beta_{2}\right)}\|Z-A\| .
$$


Then, if $\tilde{\varepsilon} \leqslant \min \left(\varepsilon_{3}, \varepsilon_{4}\right)$, (32) holds for any $A$ which satisfies that both $A$ and $A^{\prime}$ belong to $G\left(A^{*}, \tilde{\varepsilon}\right)$.

Finally, by choosing $\varepsilon=\min \left(\varepsilon_{1}, \varepsilon_{3}, \varepsilon_{4}\right)$, we complete the proof.

THEOREM 4. If the algorithm converges to a regular minimax point $A^{*}$ and $A^{*}$ is not on the boundary of $\Omega$, it converges quadratically.

Proof. Let $\left\{A_{j}, j=0,1, \ldots\right\}$ and $\left\{d_{j}, j=0,1, \ldots\right\}$ be the sequences generated by the algorithm, started with some initial $A_{0}$ and $d_{0}$. The sequence $\left\{A_{j}\right\}$ converges to $A^{*}$. We must show that if $A_{0}$ is sufficiently close to $A^{*}$, the sequence $\left\{A_{j}\right\}$ converges quadratically. We first assume that $d_{0}>\left\|\overline{A_{0}}-A_{0}\right\|_{\infty}$. This assumption will be removed later.

First suppose $A_{0}$ is close to $A^{*}$ such that all $A_{j}, j=0,1, \ldots$, belong to $G\left(A^{*}, \varepsilon\right)$ with $\varepsilon$ defined in Lemma 4 . We consider the system of equations

$$
f_{i}(W)-f_{n+1}(W)=0, \quad i=1,2, \ldots, n .
$$

It has a solution $W=A^{*}$ under the condition (a) in Definition 2. With an initial approximation $W_{0}=A_{0}$ we apply Newton's method [16] to this equation and obtain a sequence $\left\{W_{j}\right\}$ generated by the difference equations

$$
\left[\begin{array}{c}
f_{1}\left(W_{j}\right)-f_{n+1}\left(W_{j}\right) \\
\cdots \\
f_{n}\left(W_{j}\right)-f_{n+1}\left(W_{j}\right)
\end{array}\right]+\bar{J}\left(W_{j+1}-W_{j}\right)=0, \quad j=0,1, \ldots,
$$

where

$$
\bar{J}^{T}=\left[\frac{\partial}{\partial w_{1}} \cdots \frac{\partial}{\partial w_{n}}\right]^{T} \cdot\left[\begin{array}{c}
f_{1}\left(W_{j}\right)-f_{n+1}\left(W_{j}\right) \\
\cdots \\
f_{n}\left(W_{j}\right)-f_{n+1}\left(W_{j}\right)
\end{array}\right]^{T} .
$$

$\left\{W_{j}\right\}$ converges quadratically to $A^{*}$ because rank $\bar{J}=n$, which follows from

$$
\bar{J}=\left[\begin{array}{cc} 
& -1 \\
I_{n} & \vdots \\
& -1
\end{array}\right] \cdot J\left(W_{j}\right) \cdot\left[\begin{array}{c}
I_{n} \\
0 \cdots 0
\end{array}\right],
$$

where $I_{n}$ denotes the unit matrix, rank $J\left(W_{j}\right)=n+1$ for $W_{j} \in G\left(A^{*}, \varepsilon\right)$, and the elements in the last column of $J\left(W_{j}\right)$ are all the same.

To prove the theorem, we show that, if $A_{0}$ is sufficiently close to $A^{*}$ and $d_{0}>\left\|\overline{A_{0}}-A_{0}\right\|_{\infty}$, then $A_{j}=W_{j}$ for $j=0,1,2, \ldots$ We prove this by induction. Assume $W_{j}=A_{j}$ and $d_{j}>\left\|\overline{A_{j}}-A_{j}\right\|_{\infty}$ for $j=0,1, \ldots, k$. We must show that $W_{k+1}=A_{k+1}$ and $d_{k+1}>\left\|\overline{A_{k+1}}-A_{k+1}\right\|_{\infty}$.

According to Lemma $4, \overline{A_{k}}$ is determined by the intersection of the $n+1$ tangent hyperplanes of $f_{i}(A)$ at $A_{k}$ in Step 2 of the algorithm, i.e., $\overline{A_{k}}$ is determined by the equations

$$
E\left(A_{k}, x_{i}\left(A_{k}\right)\right)+\left(\nabla E\left(A_{k}, x_{i}\left(A_{k}\right)\right), \overline{A_{k}}-A_{k}\right)=\bar{e}_{k}, \quad i=1, \ldots, n+1 .
$$

Note that the equations (34) for $j=k$ are equivalent to the set of equations obtained by cancelling $\bar{e}_{k}$ in (35), since $A_{k}=W_{k}$ and $E\left(A_{k}, x_{i}\left(A_{k}\right)\right)=f_{i}\left(A_{k}\right)$. Thus, $\overline{A_{k}}=W_{k+1}$. It remains to show that $A_{k+1}=\overline{A_{k}}$ and $d_{k+1}>\left\|\overline{A_{k+1}}-A_{k+1}\right\|_{\infty}$.

Suppose that $A_{0}$ is close to $A^{*}$ such that, for all $j \geqslant 0$,

$$
\left\|W_{j+1}-A^{*}\right\| \leqslant \frac{1}{\sqrt{n}+2}\left\|W_{j}-A^{*}\right\|
$$


and $\left|e\left(W_{j+1}\right)-e\left(A^{*}\right)\right| \leqslant \frac{1}{2}\left|e\left(W_{j}\right)-e\left(A^{*}\right)\right|$. This is possible, when $A_{0}$ is sufficiently close to $A^{*}$, because $\left\{W_{j}\right\}$ converges to $A^{*}$ quadratically and the relation (30) holds. Then, applying Lemma 4, we obtain

$$
\begin{aligned}
\left|e\left(A_{k}\right)-e\left(\overline{A_{k}}\right)\right| & \geqslant\left|e\left(A_{k}\right)-e\left(A^{*}\right)\right|-\left|e\left(\overline{A_{k}}\right)-e\left(A^{*}\right)\right| \\
& \geqslant \frac{1}{2}\left|e\left(A_{k}\right)-e\left(A^{*}\right)\right| \geqslant \frac{C}{2}\left\|A_{k}-A^{*}\right\| \\
& >\frac{C}{3}\left\|A_{k}-\overline{A_{k}}\right\| \geqslant\left(1+\frac{1}{\beta_{2}}\right)\left|e\left(\overline{A_{k}}\right)-\bar{e}_{k}\right|,
\end{aligned}
$$

where the fourth inequality follows from

$$
\left\|A_{k}-A^{*}\right\| \geqslant\left\|A_{k}-\overline{A_{k}}\right\|-\left\|\overline{A_{k}}-A^{*}\right\| \geqslant\left\|A_{k}-\overline{A_{k}}\right\|-\frac{1}{\sqrt{n}+2}\left\|A_{k}-A^{*}\right\|,
$$

i.e.,

$$
\left\|A_{k}-A^{*}\right\| \geqslant \frac{\sqrt{n}+2}{\sqrt{n}+3}\left\|A_{k}-\overline{A_{k}}\right\|>\frac{2}{3}\left\|A_{k}-\overline{A_{k}}\right\| .
$$

It follows from (37) that $e\left(A_{k}\right)-e\left(\bar{A}_{k}\right)>\left(1+1 / \beta_{2}\right)\left(e\left(\bar{A}_{k}\right)-\bar{e}_{k}\right)$, i.e.,

$$
e\left(A_{k}\right)-e\left(\overline{A_{k}}\right)>\frac{\beta_{2}+1}{2 \beta_{2}+1}\left(e\left(A_{k}\right)-\bar{e}_{k}\right)>\beta_{1}\left(e\left(A_{k}\right)-\bar{e}_{k}\right),
$$

where the last step holds because, if $\beta_{2} \leqslant 1-\beta_{1}$ and $0<\beta_{1}<1$, as assumed in the algorithm, then $\left(\beta_{2}+1\right) /\left(2 \beta_{2}+1\right)>\beta_{1}$. In terms of the algorithm, (39) indicates that $A_{k+1}=\overline{A_{k}}$.

It also follows from (37) that

$$
e\left(A_{k}\right)-\bar{e}_{k}>\frac{1}{\beta_{2}}\left|\bar{e}_{k}-e\left(\overline{A_{k}}\right)\right|,
$$

which can be easily shown by separate discussions in the two different cases of $e\left(\bar{A}_{k}\right) \geqslant \bar{e}_{k}$ and $e\left(\bar{A}_{k}\right)<\bar{e}_{k}$. According to Step 4 of the algorithm, (40), then, means that

$$
\begin{aligned}
d_{k+1} & =\gamma_{2}\left\|\overline{A_{k}}-A_{k}\right\|_{\infty}>\left\|A_{k+1}-A_{k}\right\|_{\infty} \geqslant \frac{1}{\sqrt{n}}\left\|A_{k+1}-A_{k}\right\| \\
& \geqslant \frac{1}{\sqrt{n}}\left(\left\|A_{k}-A^{*}\right\|-\left\|A_{k+1}-A^{*}\right\|\right) \geqslant \frac{\sqrt{n}+1}{\sqrt{n}}\left\|A_{k+1}-A^{*}\right\| \\
& \geqslant \frac{\sqrt{n}+1}{\sqrt{n}} \cdot \frac{\sqrt{n}+2}{\sqrt{n}+3}\left\|A_{k+1}-\overline{A_{k+1}}\right\|>\left\|A_{k+1}-\bar{A}_{k+1}\right\|_{\infty},
\end{aligned}
$$

where we used (36) and (38). Note that (38) holds with $k$ replaced by $k+1$ even if $d_{k+1}$ is small, since decreasing $d_{k+1}$ will not increase $\left\|A_{k+1}-\bar{A}_{k+1}\right\|$.

Now we show that if $d_{0}=\left\|A_{0}-\bar{A}_{0}\right\|_{\infty}$ then for some $j$ we must have $d_{j}>$ $\left\|A_{j}-\overline{A_{j}}\right\|_{\infty}$. To see this, assume $d_{j}=\left\|A_{j}-\overline{A_{j}}\right\|_{\infty}$ for all $j$. We claim that there exists $\varepsilon>0$ such that $\bar{e}_{j}-e\left(A_{j}\right) \leqslant-\varepsilon d_{j}$ for all $j$ sufficiently large. Once this has been shown, arguments like those in Lemma 3 can be used to show $\left|e\left(\bar{A}_{j}\right)-\bar{e}_{j}\right| \leqslant$ $\beta_{2}\left(e\left(A_{j}\right)-\bar{e}_{j}\right)$ for all $j$ sufficiently large, so $d_{j}$ must increase at every step from some point on, and this contradiction will establish the results. Note that if 
$d_{j}=\left\|\overline{A_{j}}-A_{j}\right\|_{\infty}$ then

$$
e\left(A_{j}\right)-\bar{e}_{j} \geqslant \frac{d_{j}}{\left\|A_{j}-A_{j}^{\prime}\right\|}\left(e\left(A_{j}\right)-e_{j}\right),
$$

where $A_{j}^{\prime}$ denotes the intersection point of the tangent hyperplanes of $f_{i}\left(A_{j}\right)$, $i=1, \ldots, n+1$, at $A_{j}$, and $e_{j}=f_{i}\left(A_{j}\right)+\left(\nabla f_{i}\left(A_{j}\right), A_{j}^{\prime}-A_{j}\right)$ for any $1 \leqslant i \leqslant n+1$. To show this, let us consider $\tilde{A}_{j}=A_{j}+r\left(A_{j}^{\prime}-A_{j}\right)$, where $0<r \leqslant 1$ is a number such that $\tilde{A}_{j} \in \partial S\left(A_{j}, d_{j}\right)$. Denoting $p_{i}(A)=f_{i}\left(A_{j}\right)+\left(\nabla f_{i}\left(A_{j}\right), A-A_{j}\right), 1 \leqslant i \leqslant$ $n+1$, and $e\left(A_{j}\right)=f_{k}\left(A_{j}\right)$, we have $\bar{e}_{j} \leqslant p_{k}\left(\tilde{A}_{j}\right)\left(\right.$ since $\left.p_{k}\left(\tilde{A}_{j}\right)=\max _{i} p_{i}\left(\tilde{A}_{j}\right)\right)$ and

$$
\begin{aligned}
e\left(A_{j}\right)-\bar{e}_{j} & \geqslant e\left(A_{j}\right)-p_{k}\left(\tilde{A_{j}}\right)=p_{k}\left(A_{j}\right)-p_{k}\left(\tilde{A_{j}}\right) \\
& =\frac{\left\|A_{j}-\tilde{A}_{j}\right\|}{\left\|A_{j}-A_{j}^{\prime}\right\|}\left(p_{k}\left(A_{j}\right)-p_{k}\left(A_{j}^{\prime}\right)\right)=\frac{d_{j}}{\left\|A_{j}-A_{j}^{\prime}\right\|}\left(e\left(A_{j}\right)-e_{j}\right) .
\end{aligned}
$$

In view of (37) (proved with $A_{k}^{\prime}$ in place of $\overline{A_{k}}$ ) and (32), if $\left\|A_{j}-A^{*}\right\|$ is sufficiently small (so $\left\|A_{j}^{\prime}-A^{*}\right\|$ can also be sufficiently small, refer to the earlier parts of the proof), we have

$$
\begin{aligned}
e\left(A_{j}\right)-e_{j} & \geqslant\left|e\left(A_{j}\right)-e\left(A_{j}^{\prime}\right)\right|-\left|e\left(A_{j}^{\prime}\right)-e_{j}\right| \\
& >\frac{C}{3}\left\|A_{j}-A_{j}^{\prime}\right\|-\frac{C}{3\left(1+1 / \beta_{2}\right)}\left\|A_{j}-A_{j}^{\prime}\right\|=\frac{C}{3\left(1+\beta_{2}\right)}\left\|A_{j}-A_{j}^{\prime}\right\|
\end{aligned}
$$

and $e\left(A_{j}\right)-\bar{e}_{j}>C / 3\left(1+\beta_{2}\right) \cdot d_{j}$ for all $j$ sufficiently large. This establishes the claim. The proof of the theorem is thus completed.

COROLlaRY 2. The algorithm generates the same sequence $\left\{A_{j}\right\}$ as the one generated by the Remez second exchange method [18] in a neighborhood of a regular minimax point, provided that $F(A, x)$ is linear in $A$, with $\{F(A, x): A \in \Omega\}$ satisfying the Haar condition (i.e., no nontrivial element has more than $n-1$ zeros) on the smallest closed interval containing $\Delta$.

Indeed, in the case of linear approximation, Step 2 of the algorithm produces an $\overline{A_{j}}$ such that $E\left(\bar{A}_{j}, x\right)$ is equiripple on $\left\{x_{i}\left(A_{j}\right): i=1, \ldots, n+1\right\}$ for $\left\|A_{j}-A^{*}\right\|$ sufficiently small. This and the fact that $A_{j+1}=\bar{A}_{j}$ imply this corollary.

The unique minimax point in a polynomial or nondegenerate rational approximation is usually regular in the sense defined in this paper and, hence, our algorithm converges quadratically in these special cases. This is stated in the following theorem.

THEOREM 5. Let $R^{*}=P^{*} / Q^{*}$ be the best approximation to $f(x)$ from the class $R[\Delta]=\left\{P / Q=\left(p_{1} \Phi_{1}+\cdots+p_{s} \Phi_{s}\right) /\left(q_{1} \Psi_{1}+\cdots+q_{r} \Psi_{r}\right): Q>0\right.$ on $\left.\Delta\right\}$, where $\Phi_{1}, \ldots, \Phi_{s}, \Psi_{1}, \ldots, \Psi_{r}$ are analytic on $\Delta$. If $\left\{p_{1} \Phi_{1}+\cdots+p_{s} \Phi_{s}+\right.$ $\left.R^{*}\left(q_{1} \Psi_{1}+\cdots+q_{r} \Psi_{r}\right)\right\}$ satisfies the Haar condition on $\Delta$ with dimension $r+s-1$, condition (a) of Definition 2 is satisfied, and $\Omega$ is sufficiently large, then the algorithm converges quadratically to $R^{*}$ from an initial $R_{0}$ that is close to $R^{*}$.

Under the assumptions of the theorem, the strong unicity theorem holds [4]. Thus the condition (b) in Definition 2 is guaranteed and, consequently, the unique best approximation is regular. The algorithm has a quadratic convergence rate in a neighborhood of $R^{*}$. 
It is a conjecture that there are no other stationary points except the unique minimax point in rational or polynomial approximation. Thus the algorithm converges to the best approximation from any initial $R_{0} \in R[\Delta]$.

Combining Corollary 2 and Theorem 5, we reach the following conclusion.

COROLlaRy 3. The Remez multi-exchange method converges quadratically in the case of polynomial approximation if condition (a) of Definition 2 holds.

This well-known result (refer to [14, p. 111], where similar hypotheses were employed) is obtained here in a different way.

Finally, we have two more remarks.

If an interval in $\Delta$ reduces to be an isolated point $\tilde{x}$ (the local maximum of the approximating error on this interval is $E(A, \tilde{x})$ in view of our definition), the results in this paper still hold. Specifically, the algorithm can be applied to an approximation problem on a finite point set.

In Step 2 of the algorithm, $\left\{x_{1}, \ldots, x_{N\left(A_{k}\right)}\right\}$ could be replaced by an arbitrary finite set $\tilde{X}\left(A_{k}\right)$ containing $X_{m}\left(A_{k}\right)$, on which $\nabla E\left(A_{k}, x\right)$ exists. Then the convergence is still guaranteed. If, moreover, for all $k$ sufficiently large, (1) $X_{m}\left(A^{*}\right) \subset$ $\tilde{X}\left(A_{k}\right)$ and (2) there exists $\alpha>0$ such that if $\hat{x} \in X\left(A_{k}\right)$ and $E\left(A_{k}, \hat{x}\right) \geqslant e\left(A_{k}\right)-\alpha$ then $\hat{x} \in \tilde{X}\left(A_{k}\right)$, the quadratic convergence theorem holds. Note that the condition (2) is always satisfied when $\Delta$ is a finite point set.

5. Numerical Examples. In this section we give several simple examples to illustrate the basic features of the algorithm. These examples were run on a CYBER $170 / 730$ in double precision.

Example 1. We consider the nonlinear approximation problem discussed in [6]. The approximating function and the function to be approximated are respectively $F(A, x)=a_{1}-\left(a_{2}^{2}-x^{2}\right)^{1 / 2}$ and $f(x)=\cosh (x)-1 . \Delta$ is defined to be $[0,1]$. The difficulties of using the exchange algorithm were discussed in [6]. Applying our algorithm, with the initial $A_{0}=[1.2,1.2]$ given in [6], we obtain the following solution in four iterations: $A=[1.206907038,1.192213912]$. The maximum deviation, .014693126, is attained at $x=0, .77414215$ and 1 . Quadratic convergence was observed in this example.

Example 2. This is a problem of degenerate rational approximation given in [20]; that is, $F(A, x)=\left(a_{1}+a_{2} x\right) /\left(1+a_{3} x\right), f(x)=x^{2}, \Delta=[-1,1]$. Starting with the initial $A_{0}=[0,0,0]$, we obtained the following solution in 16 iterations, $A=$ $[.5,-.49497483,-.98994967]$. The maximum deviation .5 , is at $-1, .35 \times 10^{-14}$, and 1. In this example, to avoid the pole problem, we used $\Omega=\left\{\left(a_{1}, a_{2}, a_{3}\right):-10^{10} \leqslant a_{1}\right.$ $\left.\leqslant 10^{10},-10^{10} \leqslant a_{2} \leqslant 10^{10},-1 \leqslant a_{3} \leqslant 1\right\}$.

Example 3. It is well known that real difficulties may be encountered when a solution is almost degenerate. Let us consider an example given by Rice in [18], where $F(A, x)=\left(1+a_{1} x\right) /\left(a_{2}+a_{3} x+a_{4} x^{2}\right), f(x)=\Gamma(x)$ and $\Delta=$ [1.95, 3]. Applying the algorithm, we obtained the solution, $A=$ $[-.51282077966,2.0133138899,-1.5375272330, .25900425704]$. The maximum deviation is .0074687819 , which is attained at five points: 1.95, 1.9503960, 2.2835750, 2.8047172 and 3. This solution, with one pole at 1.9499990004 and a zero at 1.9499989854 , is nearly degenerate. Quadratic convergence was observed when $A_{j}$ was very close to $A^{*}$. The evaluation of $\Gamma(x)$ was based on the formula in [21]. 
Example 4. Consider another degenerate problem given in [15], where $F(A, x)=$ $a_{1} x+a_{2} e^{x}, f(x)=x^{2}$ and $\Delta=[0,2]$. Starting with $A_{0}=[0,0]$, after 36 iterations we obtained a solution, $A=[.18423256441, .41863121779]$, with 11 significant digits. The maximum deviation, .53824531817 , is obtained at .40634574 and 2 . Linear convergence was observed. In a degenerate case, it is expected that the convergence is slower than quadratic. It is also more difficult to obtain a highly accurate solution because of the "flat bottom" of the maximum error function. In this example, to get a solution with the above accuracy, the relative difference between $e(A)$ and $e\left(A^{*}\right)$ must be less than $10^{-22}$.

Example 5. Finally, we treat an example of polynomial approximation to verify the equivalence of the algorithm to the Remez multi-exchange method. Let $F(A, x)$ $=a_{1}+a_{2} x+a_{3} x^{2}+a_{4} x^{3}+a_{5} x^{4}, f(x)=\Gamma(x), \Delta=[2,3]$. Starting with an initial $A_{0}$, whose error was equiripple at 6 equally spaced points, the algorithm converged quadratically in 4 iterations to a solution of more than 8 significant digits. $A_{i}, i=0,1, \ldots, 4$, were exactly the same as obtained by the Remez method.

The optimization constants used for these examples were the ones given in Section 2. The choices of $d_{0}$ were not critical.

The algorithm has been successfully applied to problems of digital filter designs. This will be reported in a separate paper elsewhere. For more complicated examples refer to that paper, or [8].

Acknowledgment. The authors would like to thank an anonymous reviewer for his numerous constructive comments which served to substantially improve the mathematical theory and the presentation of the material.

Department of Radio and Electronics

University of Science and Technology of China

Hefei, Anhui province, China

Department of Electrical and Computer Engineering

State University of New York at Buffalo

Amherst, New York 14260

1. I. Barrodale, M. J. D. Powell \& F. D. K. Roberts, "The differential correction algorithm for rational $l_{\infty}$ approximation," SIAMJ. Numer. Anal., v. 9, 1972, pp. 493-504.

2. I. BarRodale \& C. Phillips, An Improved Algorithm for Discrete Chebyshev Linear Approximation, Proc. Fourth Manitoba Conf. on Numer. Math., Winnipeg, Manitoba, 1974, pp. 177-190.

3. I. BarRodale, "Linear Chebyshev approximation of complex-valued functions," Math. Comp., v. 32, 1978, pp. 853-863.

4. E. W. CheneY, Introduction to Approximation Theory, McGraw-Hill, New York, 1966.

5. Ludwig Cromme, "Eine Klasse von Verfahren zur Ermittlung bester nicht linearer TschebyscheffApproximationen,” Numer. Math., v. 25, 1975/76, pp. 447-459.

6. C. B. Dunham \& C. R. Crawford, "Minimax approximation by a semi-circle," SiaM J. Numer. Anal., v. 17, 1980, pp. 63-65.

7. W. Fraser \& J. F. Hart, "On the computation of rational approximations to continuous functions," Comm. ACM, v. 5, 1962, pp. 401-403.

8. Z. Q. Jing, Chebyshev Design of Digital Filters by a New Minimax Algorithm, Ph. D. Dissertation, State University of New York at Buffalo, 1983.

9. D. W. Kammler \& R. J. McGlinn, A Family of Fortran Programs for Finding Best Chebyshev Approximations with Applications to Exponential Sums, Research report prepared under AFOSR grant number 74-2653, Southern Illinois University, Carbondale, Ill., September 1, 1975.

10. E. H. Kaufman, JR. \& G. D. TAYLOR, "Uniform approximation with rational functions having negative poles," J. Approx. Theory, v. 23, 1978, pp. 364-378. 
11. E. H. Kaufman, JR. \& G. D. TAYloR, "An application of linear programming to rational approximation," Rocky Mountain J. Math., v. 4, 1974, pp. 371-373.

12. E. H. Kaufman, JR., D. J. Leeming \& G. D. TAYLOR, “A combined Remes-differential correction algorithm for rational approximation," Math. Comp., v. 32, 1978, pp. 233-242.

13. K. MADSEN, "An algorithm for minimax solution of overdetermined systems of nonlinear equations," J. Inst. Math. Appl., v. 16, 1975, pp. 321-328.

14. G. MeINARduS, Approximation of Functions: Theory and Numerical Methods, Springer Tracts in Natural Philosophy, Vol. 13, New York, 1967.

15. M. R. Osborne \& G. A. WATSON, "A note on singular minimax approximation problems," $J$. Math. Anal. Appl., v. 25, 1969, pp. 692-700.

16. G. M. Phillips \& P. J. TAYLOR, Theory and Applications of Numerical Analysis, Academic Press, New York, 1973.

17. M. J. D. Powell, The Minimax Solution of Linear Equations Subject to Bounds on the Variables, Proc. Fourth Manitoba Conf. on Numer. Math., Winnipeg, Manitoba, 1974, pp. 53-107.

18. J. R. Rice, The Approximation of Functions, vol. 2, Addison-Wesley, Reading, Mass., 1969.

19. JOHN A. ROULIER \& G. D. TAYLOR, "Uniform approximation by polynomials having bounded coefficients," Abh. Math. Sem. Univ. Hamburg, v. 36, 1971, pp. 126-135.

20. G. A. WATSON, "A method for calculating best nonlinear Chebyshev approximations," J. Inst. Math. Appl., v. 18, 1976, pp. 351-360.

21. H. Werner \& R. Collinge, “Chebyshev approximation to the gamma function," Math. Comp. v. 15, 1961, pp. 195-197. 